\title{
BMJ Open Quality More than pills: alternative adjunct therapies to improve comfort in hospitalised patients
}

Megan Moore, ${ }^{1}$ Maria Schuler, ${ }^{1}$ Samantha Wilson, ${ }^{1}$ Morgan Whisenhunt, ${ }^{1}$ Ashleigh Adams, ${ }^{1}$ Britany Leiker, ${ }^{1}$ Tori Butler, ${ }^{1}$ Caylin Shankweiler, ${ }^{2}$ Matthew Jones, ${ }^{2}$ Cheryl Gibson ${ }^{3}$

To cite: Moore M, Schuler M, Wilson S, et al. More than pills: alternative adjunct therapies to improve comfort in hospitalised patients. BMJ Open Quality 2019;8:e000506. doi:10.1136/ bmjoq-2018-000506

Received 14 August 2018 Revised 15 March 2019 Accepted 22 April 2019

\section{Check for updates}

C Author(s) (or their employer(s)) 2019. Re-use permitted under CC BY-NC. No commercial re-use. See rights and permissions. Published by BMJ.

${ }^{1}$ NS 62-Medical Telemetry, The University of Kansas Health System, Kansas City, Kansas, USA

${ }^{2}$ Internal Medicine, The University of Kansas Health System, Kansas City, Kansas, USA

${ }^{3}$ Internal Medicine, The University of Kansas Medical Center, Kansas City, Kansas, USA

Correspondence to

Caylin Shankweiler,

cshankweiler@kumc.edu

\section{ABSTRACT}

Discomfort in the hospitalised patient continues to be one of the healthcare system's greatest challenges to positive patient outcomes. The patients' ability to focus on healing is impaired by discomforts such as pain, nausea and anxiety. Alternative, non-pharmacological therapies have shown to be effective in reducing discomfort and managing pain, complementing analgesic agents and optimising pain therapy modalities. This multi-cycle project is aimed to assess the effect of alternative therapies on inpatient, progressive care patients who reported discomfort or little to no relief in discomfort from prescribed analgesics and adjuvant agents. In the first Plan Do Study Act (PDSA) cycle, patients who reported discomfort were offered aromatherapy or visual relaxation DVDs. In the second PDSA cycle, patients were offered a comfort menu that consisted of multiple alternative interventions such as aromatherapy, ice or heat and ambulation. During each cycle, participants completed a survey measuring comfort levels before and after patientselected alternative intervention(s) were administered. In the first PDSA cycle, $88 \%$ of patients reported an increase in comfort level after the intervention, and $97 \%$ reported an interest in using alternative therapy again. In the second PDSA cycle, $47 \%$ reported increased comfort, and $89 \%$ indicated a willingness to try alternative therapies again for improvement of comfort level. Overall, the quality improvement project increased the level of comfort reported by hospitalised patients, creating a gateway to comfort with less emphasis on prescribed analgesic medications.

\section{PROBLEM}

Many times, the causes of discomfort for hospitalised patients are complex, and effective ways to reduce pain, anxiety, stress and nausea without the sole use of pharmacological interventions are needed. Although the use of non-pharmacological methods to maximise pain relief has been demonstrated, these methods have not been offered routinely as a first-line intervention. ${ }^{1-4}$ The purpose of this study is to implement a process that supports the utilisation of alternative methods such as aromatherapy, visual relaxation DVDs and the application of ice or heat to decrease the causes of patient discomfort while simultaneously addressing the standards of care for their medical diagnosis.

This quality improvement project was performed at the University of Kansas Health System, a regional academic medical centre providing a full range of inpatient services in the greater Kansas City area. The project was conducted on a 22-bed medical telemetry unit that primarily cares for internal medicine patients with infection, gastrointestinal, hepatobiliary and cardiac disorders. To understand how well patients' reports of pain were addressed before the initiation of the quality improvement project, team members examined patient ratings of pain intensity, using the Numeric Pain Rating Scale (NPRS)..$^{5}$ The NPRS is an 11-point scale, from 0 to 10 , indicating no pain to the most intense pain imaginable. Many patients reported experiencing little to no change in pain intensity after pain medication was administered. In response, the team explored alternative interventions that could better aid in pain management and sought effective comfort therapies to reduce distressful symptoms such as nausea and anxiety.

\section{BACKGROUND}

During the 1990s, pain was accepted as a 'fifth vital sign' that highlighted the need for improved pain management and supported the notion that pain was undertreated. ${ }^{7-9}$ During that period, a push to enact policies to control and manage patients' pain levels was initiated on a national level. Organisations such as the Veteran's Health Association and the Joint Commission quickly accepted the policy standards for the management of pain and introduced a national strategy to improve pain treatment. ${ }^{10}$ Prescriptions for pain medication by healthcare providers increased substantially. Inadvertently, providers began prescribing analgesic medications that have 
addictive properties to patients who complained of pain, which many believe has led to the overtreatment and overuse of opioids. ${ }^{2}$ According to the Centers for Disease Control and Prevention, approximately 115 Americans die every day from an opioid overdose,${ }^{11}$ and prescription opioids have been confirmed to be a large contributor to the opioid epidemic. ${ }^{12} 13$

In response to public outcry of opioid-related deaths, hospitals have implemented action alerts and educational sessions to reduce the number of opioid prescriptions while managing patients' chronic discomfort. ${ }^{14} 15$ However, the inpatient setting continues to have patients who suffer from acute and chronic pain, stress, anxiety, nausea and other discomforting symptoms. Managing these symptoms, while reducing the risk for future potential addictions, has become a priority for healthcare providers. Alternative therapies to relieve pain and enhance comfort such as aromatherapy, visual relaxation DVDs and ice or heat have the capacity to provide a way for discomfort to be addressed in a safe manner. ${ }^{16-18}$ Aromatherapy has proven to decrease stress, depression and anxiety. ${ }^{19}$ Further, findings reported in a systematic review support the use of music as an adjuvant approach to pain control in adult hospitalised patients. ${ }^{20}$ Moreover, using non-pharmacological interventions to address comfort without the adverse effects associated with opioid use can improve patient safety and satisfaction with their perception of comfort and pain relief.

\section{MEASUREMENT}

Before an alternative pain therapy reduction method was selected by the patient, nurses asked patients to rate their comfort level, using the Clinically Aligned Pain Assessment Tool (CAPA), a four-item scale ranging from intolerable, tolerable with discomfort, comfortably manageable, to negligible discomfort. ${ }^{21}$ The decision was made to switch from the NPRS to the CAPA tool to guide administration for nurses, prompting better conversation with patient about their discomfort. Thus, allowing the patient to specify their discomfort beyond a number. Patients were asked to indicate what type of discomfort they were experiencing (ie, pain, anxiety, nausea, insomnia or other discomfort). When a patient reported discomfort, they were offered a list of alternative therapy options from which to choose. In cycle 1 , alternative therapy options included aromatherapy and visual relaxation DVDs. In cycle 2, the options included alternative methods as displayed in online supplementary appendix 1. One-hour post alternative therapy, patients were asked to re-assess their comfort level, using the same CAPA tool, and to report how likely they were to use the therapy again during times of discomfort. In the first cycle, sleep was noted but not rated using the CAPA tool. In the second cycle, if a patient was sleeping during post assessment, then the nurse noted this as 'negligible discomfort' on the assessment form. Other researchers have indicated improving patient sleep represents a natural starting point for improving comfort. ${ }^{22}$ Nurses also tracked how many patients refused alternative therapy.

All patients who participated in alternative methods for pain and discomfort reduction had the potential to be surveyed more than once (ie, each day throughout their hospital stay). This was allowed due to factors such as an extended length of stay, different procedures completed throughout the patient's stay and change in a patient's condition during his/her admission or readmission.

\section{DESIGN}

It was evident that the unit needed a standardised process to provide patients with appropriate alternative therapies. The project team consisted of content experts, frontline nursing staff and unit management. The team members met monthly and intermittently engaged with the hospital's pain management team. The goal was to provide alternative comfort measures for patients who reported discomfort or who were already receiving pain relief medication but reported continued discomfort. The stakeholders agreed that the CAPA tool would be used to determine patients' discomfort levels, and as a method by which alternative therapy was provided consistently. Any patient who reported lack of relief from discomfort was eligible to participate in the quality improvement project and was offered alternative therapies. Discomfort was defined by the patient's perception of anxiety, pain, restlessness and irritability. The implementation of aromatherapy and visual relaxation DVDs was standardised across unit clinical staff. To support inter-professional collaboration, rounding providers were informed about the study and the methods of implementation. Additionally, nursing staff was educated on the process, and onboarding of new staff included orientation from key stakeholders.

\section{STRATEGY}

The aim of this quality improvement project was to increase comfort in hospitalised patients on a medical telemetry unit by using non-pharmacological interventions. The study spanned two PDSA test cycles.

PDSA cycle 1: In this PDSA cycle, the effectiveness of two alternative therapies, aromatherapy and visual relaxation DVDs, was tested. The patient was surveyed before and 1 hour post implementation of the alternative therapy. At the conclusion of this cycle, patients and frontline providers suggested the need for additional alternative therapies. Based on feedback, the project team collaborated with content experts to expand alternative therapy options available to patients.

PDSA cycle 2: During this cycle, the team offered additional alternative therapies to achieve a higher level of comfort among the patient population. A Comfort Menu was developed (see online supplementary appendix 1) with explanations of each intervention to help patients visualise options. For this cycle, two different nurses (one who was involved in the intervention and one who was not) 
surveyed the patient before and after the intervention to reduce potential nurse bias. The nurse who brought the patient the alternative therapy completed the initial survey. One hour after the intervention had been implemented, a different nurse asked the patient the same survey questions. Outcomes from this cycle established the need for hospital-wide implementation. Additionally, continued use of the Comfort Menu has been sustained through onboarding of new frontline providers.

\section{RESULTS}

The main outcome measure was comfort level post alternative therapies for patients reporting discomfort on the inpatient telemetry unit. In cycle 1, 104 surveys were administered to 65 unique patients over a 12-week period. Of those patients, $25 \%$ were admitted with the common diagnosis of infection, and the most commonly reported discomfort type was pain $(37 \%)$, followed by a combination of discomfort (ie, pain and anxiety) at $35 \%$. In this cycle, $77 \%$ of those who were offered alternative therapy agreed to partake, and $2 \%$ of those who partook reported negligible discomfort, $25 \%$ reported being comfortably manageable, $60 \%$ reported tolerable with discomfort and $12 \%$ reported intolerable discomfort at the time of intervention. One hour post intervention, comfort was reassessed: $53 \%$ were sleeping on reassessment, $15 \%$ reported negligible discomfort, $21 \%$ reported being comfortably manageable, $10 \%$ reported tolerable with discomfort and $1 \%$ reported intolerable discomfort. For cycle 1, the outcome represented an $89 \%$ improvement in comfort level from the baseline assessment (see figure 1). Secondary to assessing comfort level, the project team asked if the patient would use the therapy again. Ninety-eight percent of respondents indicated positively about using alternative therapy to enhance comfort.

In cycle 2, the team broadened the approach to alternative therapy with the Comfort Menu. Change in the level of discomfort and control of comfort as reported by the patient was tracked. One hundred and one surveys were administered to 78 unique patients. Similar to the first cycle, the most common admission diagnosis was infection $(21 \%)$, and the most common reported discomfort type was pain $(78 \%)$. In cycle $2,76 \%$ of patients agreed to participate in alternative comfort therapies, and the most commonly selected alternative therapy methods are displayed in figure 2. At the time of the intervention, 3\% of the patients reported negligible discomfort, $12 \%$ were comfortably manageable, $59 \%$ reported tolerable with discomfort and $27 \%$ had intolerable discomfort. One hour post intervention, $6 \%$ stated negligible discomfort, $42 \%$ of patients reported being comfortably manageable, $40 \%$ reported tolerable with discomfort and $13 \%$ had intolerable discomfort. For this cycle, the outcome represented a $47 \%$ improvement in comfort from the baseline assessment. Additionally, when patients were asked how they perceived their change in comfort, $51 \%$ of

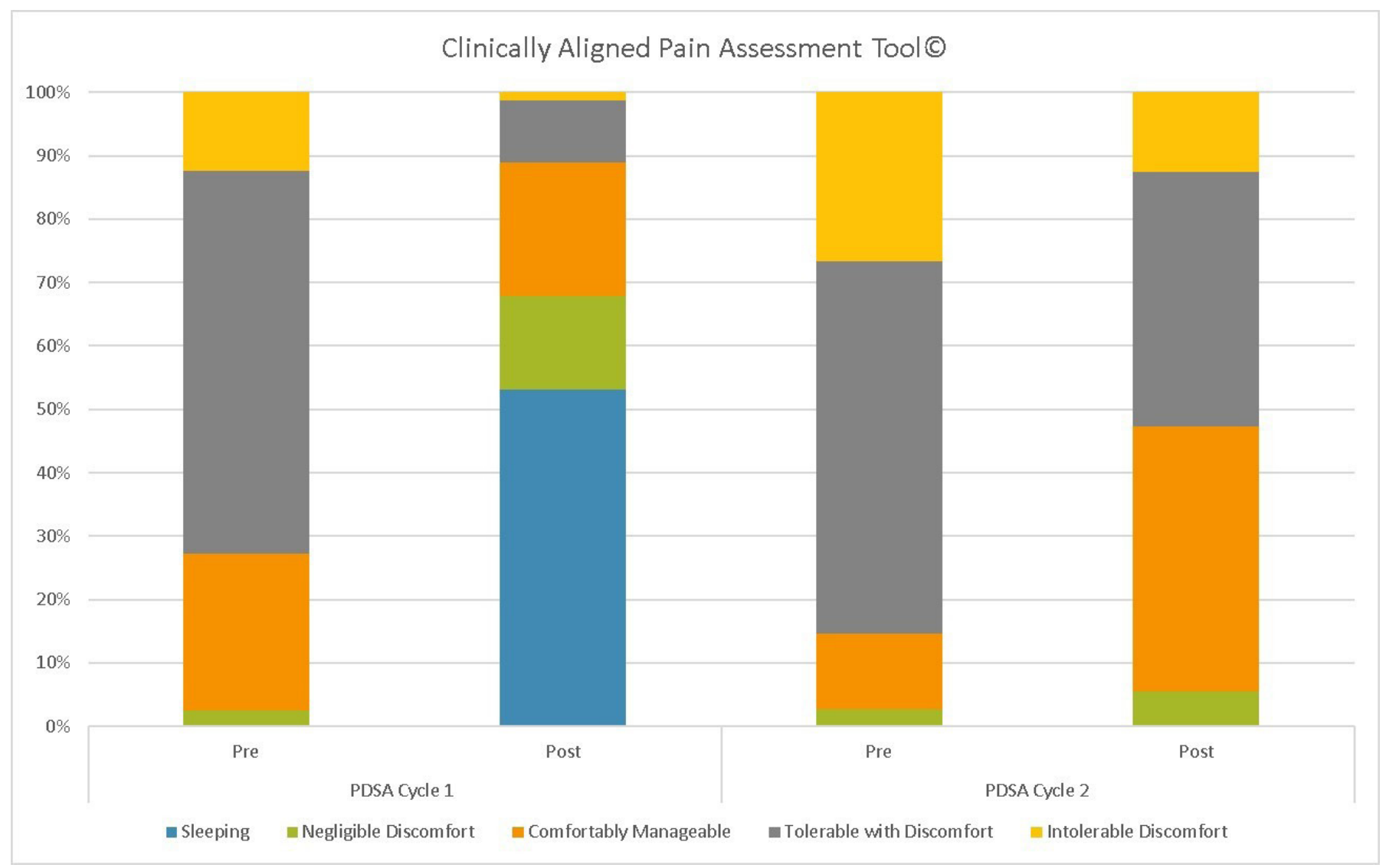

Figure 1 Clinically aligned pain assessment tool results for cycle 1 and cycle 2. PDSA, Plan Do Study Act. 


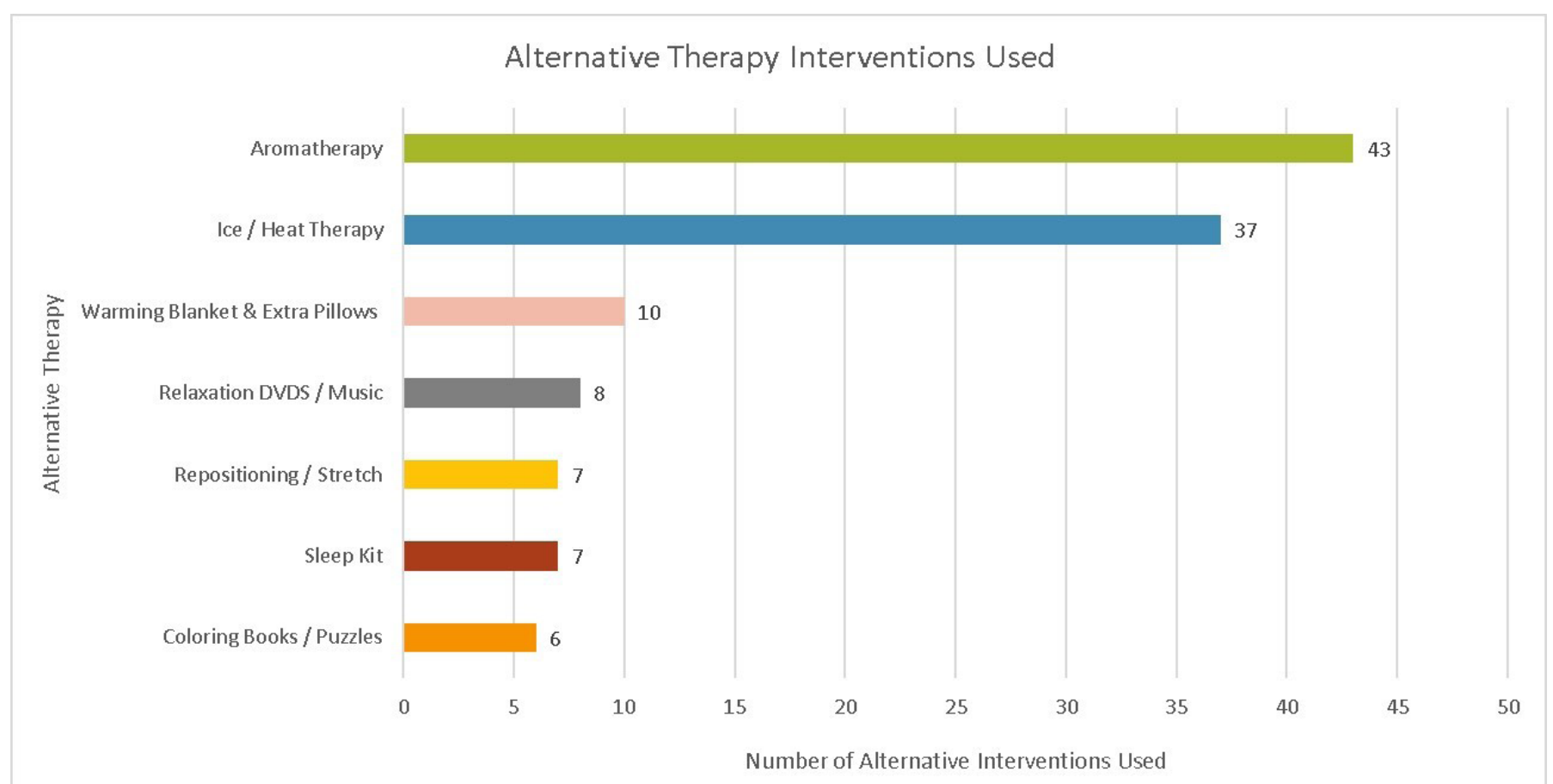

Figure 2 Most commonly selected alternative therapies from PDSA cycle 2. *Patients had the option to select more than one intervention. PDSA, Plan Do Study Act.

patients reported their discomfort as 'getting better', and $70 \%$ stated the comfort menu options were effective in controlling discomfort. Furthermore, $89 \%$ of those who tried alternative pain interventions would do so again.

\section{LESSONS AND LIMITATIONS}

Patients might have been surveyed more than once because there was no effective way of tracking whether or not a patient had been surveyed previously by another nurse. The survey instrument was also adapted to include the option 'sleeping' in the first cycle; however, this was not included in the second cycle because it was considered 'negligible discomfort'. Lastly, there was inconsistent use of specific brands of aromatherapy oil throughout the study.

Similar to the first cycle's limitation, the second cycle had no effective method of tracking if a patient had been surveyed previously by another nurse. Another challenge was keeping the visual relaxation DVDs consistently available because items were lost or inadvertently taken home by patients. Furthermore, there was also a limitation of the inability to stock and source aromatherapy oils in house to ensure supply consistency. This inconsistency was related to the aromatherapy oils becoming available across all hospital units. The project team attempted to notify materials management of this house-wide expansion in advance but the oils were placed on backorder.

In both cycles, patients were initially reluctant to participate in alternative therapies instead preferring pharmacological treatment options because of their unfamiliarity and lack of exposure to non-pharmacological therapies. However, in the second cycle, the team benefited from training provided by specialists in relaxation and alternative discomfort relief on how to discuss alternative therapy options with patients. Additionally, a visual menu proved helpful for patients when selecting potential non-pharmacological interventions and enhancing exposure towards alternative therapies. Finally, our project team recognised that non-pharmacological comfort measures were not always available to the patient following discharge from the hospital, and further data are needed on patients' ability to sustain use of alternative therapy post discharge.

\section{CONCLUSION}

The multi-cycle QI project was successful in relieving patient discomfort by providing alternative therapies to inpatient, progressive care patients who reported little to no relief in discomfort from prescribed analgesics and adjuvant agents. Bridging off the success of the first cycle, a Comfort Menu with a greater variety of pain relief options was created, successfully implemented and well received by patients. The pain relief options were offered at no cost to the patient as most of the tools and supplies used were donated or covered in the hospital unit budget. Patients demonstrated tolerance of their discomfort when using alternative modalities and were highly likely to use these alternative comfort therapies again. This project has already gone through two PDSA cycles and has proven sustainability. Ten months post cycle 2 , aromatherapy was implemented across all units of the hospital with support via development of a procedure. After the completion of cycle 2, external grant funding was awarded to support both the continuation of unit-based comfort offerings and the maintenance of the project. Future efforts will be 
focused on decreasing pharmacological therapies for the relief of pain and evaluating the effectiveness of pharmacological interventions in the relief of patient discomfort. Additionally, efforts will be aimed at implementing the use of all Comfort Menu interventions throughout the health system. Processes will be developed to partner with prescribing providers to integrate these alternative treatment modalities into standard practice.

In summary, this project is timely as supported by a highly publicised opioid epidemic in this country. In a period where federal government officials are providing stepwise approaches to pain control, continued evaluation and more projects like this one are pivotal in having impact on this epidemic. This project demonstrates successful implementation of alternative therapies and suggests non-pharmacological treatments for pain and discomfort can be an integral part of a comprehensive treatment approach. ${ }^{23}$ Future studies should further evaluate alternative therapies as an adjuvant to pharmaceutical, physical and exercise therapy, and cognitive behavioural therapy, especially in response to the Surgeon General's call to end the opioid epidemic. ${ }^{24}$

Acknowledgements Preliminary results were accepted as an abstract at the Annual Conference of the Vizient/AACN Nurse Residency Program held in March 2017, the RNovator KC 31st Annual Visions Symposium held in November 2017 and the 31st Annual Visions Symposium held in March 2018. The authors thank Melanie Simpson, Sara Hocking, Jennifer Surprise, Noreen Thompson, Caroline Black Jenn Ray, Sarah Larson and Moira Mulhern for their invaluable feedback and support for this project.

Contributors MM, MS, SW, MW, AA, BL and TB made substantial contributions to the conception and design, acquisition of data, analysis and interpretation of data. All authors drafted and revised the manuscript for important intellectual content. $\mathrm{CS}, \mathrm{CG}$ and MJ made substantial contributions to the submitted manuscript by assisting with the acquisition of data, analysis and interpretation of data, drafting of the manuscript, statistical analyses and critical revisions of the manuscript. All authors read and approved the final manuscript.

Funding This research received no specific grant from any funding agency in the public, commercial or not-for-profit sectors.

Competing interests None declared.

Patient consent for publication Not required.

Ethics approval The quality improvement protocol was approved by the Institutional Review Board of the University of Kansas Medical Center.

Provenance and peer review Not commissioned; externally peer reviewed.

Open access This is an open access article distributed in accordance with the Creative Commons Attribution Non Commercial (CC BY-NC 4.0) license, which permits others to distribute, remix, adapt, build upon this work non-commercially, and license their derivative works on different terms, provided the original work is properly cited, appropriate credit is given, any changes made indicated, and the use is non-commercial. See: http://creativecommons.org/licenses/by-nc/4.0/.

\section{REFERENCES}

1. Jonas WB, Eisenberg D, Hufford D, et al. The evolution of complementary and alternative medicine (cam) in the USA over the last 20 years. Forsch Komplementmed 2013;20:65-72. 2006.

2. Alexander GC, Kruszewski SP, Webster DW. Rethinking opioid prescribing to protect patient safety and public health. JAMA 2012;308:1865-6.

3. Kwekkeboom KL, Bratzke LC. A systematic review of relaxation, meditation, and guided imagery strategies for symptom management in heart failure. J Cardiovasc Nurs 2016;31:457-68.

4. Kwekkeboom KL, Gretarsdottir E. Systematic review of relaxation interventions for pain. J Nurs Scholarsh 2006;38:269-77.

5. Williamson A, Hoggart B. Pain: a review of three commonly used pain rating scales. J Clin Nurs 2005;14:798-804.

6. Ferreira-Valente MA, Pais-Ribeiro JL, Jensen MP. Validity of four pain intensity rating scales. Pain 2011;152:2399-404.

7. Tompkins DA, Hobelmann JG, Compton P. Providing chronic pain management in the "Fifth Vital Sign" Era: Historical and treatment perspectives on a modern-day medical dilemma. Drug Alcohol Depend 2017;173 Suppl 1(Suppl 1):S11-S21.

8. Mularski RA, White-Chu F, Overbay D, et al. Measuring pain as the 5 th vital sign does not improve quality of pain management. $J$ Gen Intern Med 2006;21:607-12.

9. Quality improvement guidelines for the treatment of acute pain and cancer pain. American pain Society quality of care Committee. JAMA 1995;274:1874-80.

10. Directive VHA, Directive VHA, Department of Veteran Affairs. Pain Management. In: VHA Directive 2009-053, ed, 2009.

11. Centers for Disease Control and Prevention. Opioid overdose: understanding the epidemic. Available: https://www.cdc.gov/ drugoverdose/epidemic/index.html2018 [Accessed Feb 2018].

12. Dart RC, Surratt HL, Cicero TJ, et al. Trends in opioid analgesic abuse and mortality in the United States. N Engl J Med 2015;372:241-8.

13. Paulozzi $L$, Ryan G. Opioid analgesics and rates of fatal drug poisoning in the United States. Am J Prev Med 2006;31:506-11.

14. Weiner SG. Changing dispensers may prevent intoxication from isopropanol and ethyl alcohol-based hand sanitizers. Annals of Emergency Medicine 2007;50.

15. Osborn SR, Yu J, Williams B, et al. Changes in provider prescribing patterns after implementation of an emergency department prescription opioid policy. J Emerg Med 2017;52:538-46.

16. Burhenn P, Olausson J, Villegas G, et al. Guided imagery for pain control. Clin J Oncol Nurs 2014;18:501-3.

17. Charette S, Fiola JL, Charest M-C, et al. Guided imagery for adolescent post-spinal fusion pain management: a pilot study. Pain Manag Nurs 2015;16:211-20.

18. Carlson AH. Hot \& cold. Tried and true ice and heat modalities still prove effective for acute and chronic pain. Rehab Management 2007;20:32-3.

19. Tang SK, Tse MYM. Aromatherapy: does it help to relieve pain, depression, anxiety, and stress in community-dwelling older persons? Biomed Res Int 2014;2014:1-12.

20. Cole LC, LoBiondo-Wood G. Music as an adjuvant therapy in control of pain and symptoms in hospitalized adults: a systematic review. Pain Manag Nurs 2014;15:406-25.

21. Topham D, Drew D. Quality improvement project: replacing the numeric rating scale with a clinically aligned pain assessment (CAPA) tool. Pain Manag Nurs 2017;18:363-71.

22. Vincent J-L, Shehabi Y, Walsh TS, et al. Comfort and patient-centred care without excessive sedation: the eCASH concept. Intensive Care Med 2016;42:962-71.

23. Stanos S, Brodsky M, Argoff C, et al. Rethinking chronic pain in a primary care setting. Postgrad Med 2016;128:502-15.

24. Murthy VH. Ending the Opioid Epidemic - A Call to Action. N Engl $J$ Med 2016;375:2413-5. 